\title{
Fatal Pediatric Motor Vehicle Crashes on U.S. Native American Indian Lands Compared to Adjacent Non-Indian Lands: Restraint Use and Injury by Driver, Vehicle, Roadway and Crash Characteristics
}

\author{
Shin Ah Oh ${ }^{1}$, Chang Liu ${ }^{2}$ and Joyce C. Pressley ${ }^{2,3,4, *}$ \\ 1 Department of Biostatistics, Columbia University, 722 West 168th Street, New York, NY 10032, USA; \\ so2461@cumc.columbia.edu \\ 2 Department of Epidemiology, Columbia University; 722 West 168th Street, New York, NY 10032, USA; \\ cl3321@cumc.columbia.edu \\ 3 Department of Health Policy and Management, Columbia University, 722 West 168th Street, New York, \\ NY 10032, USA \\ 4 Center for Injury Epidemiology and Prevention, Columbia University, 722 West 168th Street, New York, \\ NY 10032, USA \\ * Correspondence: jp376@cumc.columbia.edu; Tel.: +1-212-342-0421
}

Received: 15 August 2017; Accepted: 13 October 2017; Published: 25 October 2017

\begin{abstract}
There are large disparities in American Indian pediatric motor vehicle (MV) mortality with reports that several factors may contribute. The Fatality Analysis Reporting System for 2000-2014 was used to examine restraint use for occupants aged 0-19 years involved in fatal MV crashes on Indian lands $(n=1667)$ and non-Indian lands in adjacent states $(n=126,080)$. SAS GLIMMIX logistic regression with random effects was used to generate odds ratios (OR) with $95 \%$ confidence intervals (CI). Restraint use increased in both areas over the study period with restraint use on Indian lands being just over half that of non-Indian lands for drivers (36.8\% vs. $67.8 \%, p<0.0001)$ and for pediatric passengers $(33.1 \%$ vs. $59.3 \%, p<0.0001)$. Driver restraint was the strongest predictor of passenger restraint on both Indian and non-Indian lands exerting a stronger effect in ages 13-19 than in 0-12 year olds. Valid licensed driver was a significant predictor of restraint use in ages $0-12$ years. Passengers in non-cars (SUVs, vans and pickup trucks) were less likely to be restrained. Restraint use improved over the study period in both areas, but disparities failed to narrow as restraint use remains lower and driver, vehicle and crash risk factors higher for MV mortality on Indian lands.
\end{abstract}

Keywords: motor vehicle crash; Indian lands; restraint use

\section{Introduction}

Motor vehicle (MV) crashes are the leading cause of unintentional injury in the U.S. for ages 0 to 19 years [1]. Racial and ethnic disparities exist in MV-related mortality, with American Indians aged 0 to 19 years having higher mortality [2]. Historical trends have generally shown maintenance or widening of these disparities despite a lowering of the actual mortality rate in all race and ethnic groups [3].

This occurred as MV mortality rates fell in all race and ethnic groups, but with smaller improvements for American Indian/Alaskan Natives that produced an actual widening of MV occupant disparities [3]. Appropriate restraining of children in motor vehicles is effective in lowering child MV crash mortality [4-6], but American Indian pediatric passengers are reported to be less likely to be properly restrained [3,7-10], thus potentially increasing the risk of mortality. Several risk factors for increased risk of injury and mortality among American Indians have been identified [11-15], 
although there are also reports of racial misclassification for American Indians [16-18]. In this study, we avoid some of the potential bias that could be introduced by racial misclassification of American Indians by examining whether the crash occurred on federally-designated Indian lands or nearby lands that were not designated as such. Indian lands have a higher percentage of single-vehicle fatal crashes that have been associated with increased likelihood of fatality [15]. Within those geographic categories, we report race and ethnicity, although our multivariable models do not depend on racial classifications.

This study examines fatal motor vehicle crashes involving transport of a pediatric passenger in states with federally recognized Indian lands and compares crashes occurring on Indian lands to those occurring on non-Indian lands in the states that contain these Indian lands. In particular, among a pediatric population aged 0-19 years involved in a fatal MV crash, we examine: (1) predictors of restraint use on Indian lands compared to non-Indian lands by age, driver and vehicle characteristics, and (2) crash, environmental and roadway characteristics on Indian lands and non-Indian lands.

\section{Materials and Methods}

\subsection{Data Source}

The data used in this study is from the Fatality Analysis Reporting System (FARS) (2000-2014) publicly released by National Highway Traffic Safety Administration (NHTSA) [19]. FARS is a nationwide census of fatal injuries in MV traffic crashes on U.S. public roads and contains variables that characterize person, vehicle and crash factors.

\subsection{Study Population}

The study was limited to the following U.S. states that contain federally designated Indian lands: Alabama, Arizona, California, Colorado, Connecticut, Florida, Idaho, Indiana, Iowa, Kansas, Louisiana, Maine, Massachusetts, Michigan, Minnesota, Mississippi, Montana, Nebraska, Nevada, New Mexico, New York, North Carolina, North Dakota, Oklahoma, Oregon, Rhode Island, South Carolina, South Dakota, Texas, Utah, Virginia, Washington, Wisconsin, and Wyoming [20]. Of the 957,223 crashes in the states with federally designated Indian lands, 127,747 (13.3\%) crashes were to passenger vehicles transporting an infant, child or teen passenger (Figure 1). 


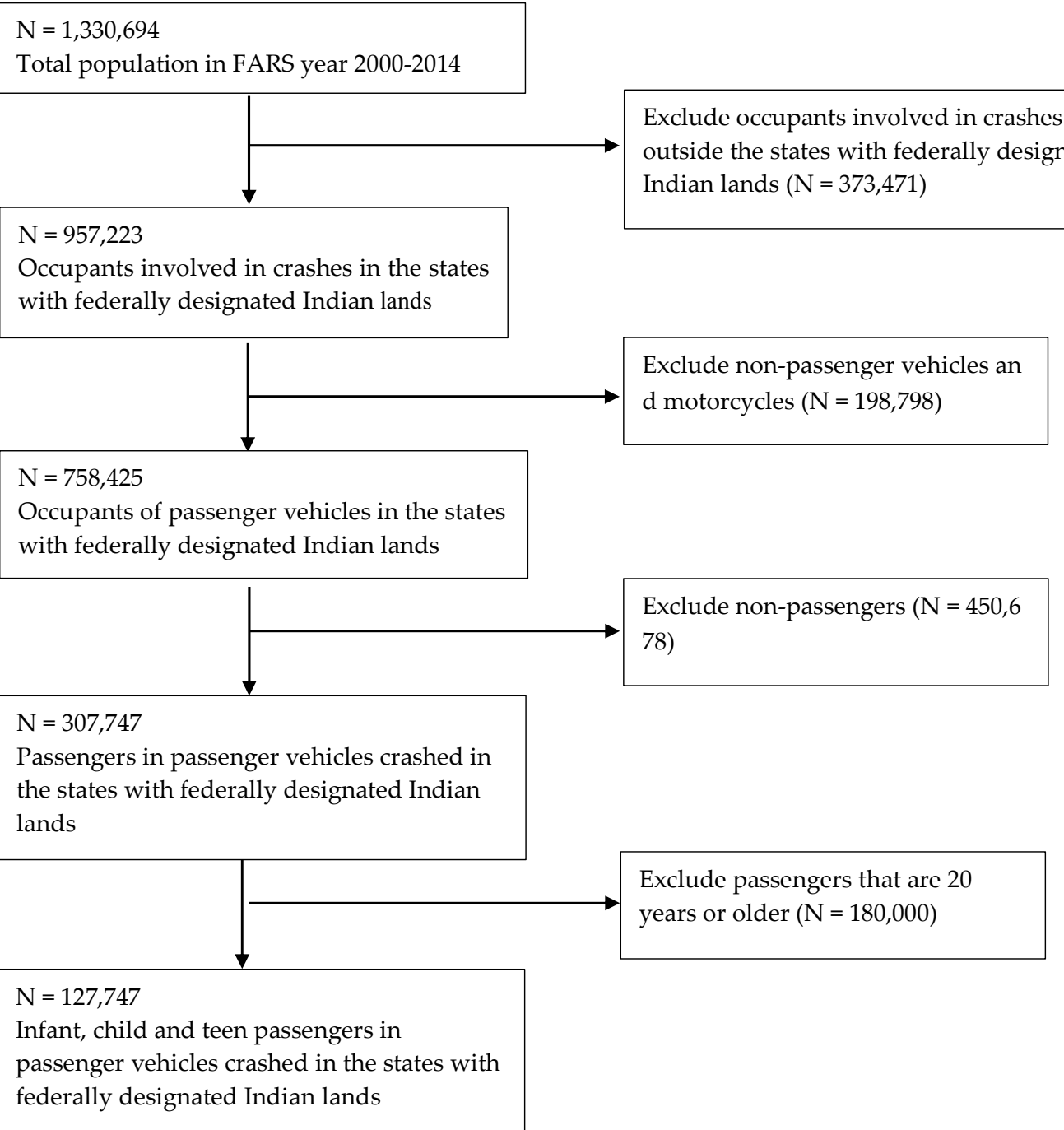

Figure 1. Population flow diagram of the study population of passengers aged 0-19 years, FARS 2000-2014.

\subsection{Variable Classification}

\subsubsection{Person-Level Characteristics}

- Passenger Restraint Status (Outcome). This is a dichotomous variable, restrained or not restrained, with use of any type of restraint being categorized as restrained. Improper restraint use or inappropriate restraint use for the age of the child were not examined outside of the restraint category.

- Passenger Mortality. This was categorized dichotomously from injury severity data in FARS. If the passenger was known to have a fatal injury the passenger was categorized as having died.

- Passenger Age and Gender. Passenger age was categorized as follows: 0-2, 3-8, 9-12, 13-14, 15-17, and 18-19 years. Gender was characterized as male or female.

- Passenger Seating Position. This was comprised of 5 categories-front, rear right or left side, rear middle, other or unknown. Passengers travelling in the sleeper section of the vehicle, cargo area or beyond fifth row of seats were categorized as 'other'.

- Driver Age and Gender. Driver age was categorized for the 76,428 drivers as follows: less than 20 years old, 20-44, 45-64, and 65 years or older. Gender was characterized as male or female. 
- Driver License Validity. License was considered valid at the time of the crash if the driver had a valid learner's permit, intermediate or full license or a temporary license. A driver's license was considered invalid if it had been suspended, revoked, expired, or cancelled at the time of crash.

- Driver Drug or Alcohol Status. This variable was categorized as negative, positive or not tested. A positive status included any of the following: (1) Police reported alcohol involvement; (2) driver blood alcohol concentration greater than or equal to 0.01 ; or (3) the driver otherwise had a positive drug or alcohol test result. If the driver didn't match any of these criteria and had no missing data in any of these variables, then the driver was categorized as negative. If the records from all those variables indicate that the driver was not tested, then the driver was categorized as not tested and alcohol and drug status as unknown/missing.

- Driver Previous Moving Violation. This was a dichotomous variable of yes or no based on having received a citation within 3 years of the crash date for previously driving while intoxicated, speeding or another moving violation.

- Driver Race and Ethnicity in Death Certificate. Driver race was categorized as White, non-Hispanic; Black, non-Hispanic, Hispanic, White or Black; Native American; Asian or Pacific Islander; and Multiple/other. Race and ethnicity were available only for occupants who died.

\subsubsection{Vehicle-Level Characteristics}

- Vehicle Model Year. Vehicle model year was examined as a categorical variable $(<1994,1994-1997$, 1998-2004, 2005-2008, 2009-2011, 2012-2014) [21].

- Vehicle Model Type. Vehicle type was categorized as passenger car, utility vehicle (SUV), van or pickup truck. Vehicles such as large trucks, motorcycles and buses were excluded.

\subsubsection{Crash-Level Characteristics}

- Day/night. Day was defined as 6:00 a.m. to 5:59 p.m. and night was defined as 6:00 p.m. to 5:59 a.m.

- Weekday/weekend. Social weekend was defined as Friday at 5:00 p.m. to Sunday at 4:59 p.m., and times outside this were categorized as weekday [22].

- Rollover or Ejection. If the vehicle and the occupants experienced either rollover or ejection or both, the data was categorized as having had rollover or ejection.

- Manner of Collision. Manner of collision was analyzed by categorizing the collision types as rear-end, head-on, angle, sideswipe or other. Non-collision was used as a category for the crashes that did not involve collision with motor vehicle in transport.

\subsubsection{Road Characteristics}

- Number of Lanes. Number of lanes was categorized as one, two, three, four, five and six or more.

- Trafficway. The categories for this variable were one-way; two-way, divided; two-way, not divided; and other. Both unprotected median and positive median barriers were considered divided.

- Route Signing. This variable was categorized in FARS as interstate, highway, country road, local street or other.

- Traffic Devices and Signs. This was categorized as no controls, traffic signals, regulatory signs or other.

- Traffic Control Device Functioning. Three categories were used-not functioning properly, functioning properly and no controls.

\subsection{Statistical Analysis}

Chi-square tests were used to assess the associations between the geographic area (Indian land vs. non-Indian land) status of crash location and potential covariates. Significance was defined as having a $p$-value of less than or equal to 0.05. PROC GLIMMIX in SAS with random effects was 
used to generate the odds ratios and 95\% confidence intervals for Indian lands and non-Indian lands, to account for the effects of clustering by multiple passengers in the same vehicle. Separate models were constructed for Indian lands and non-Indian lands to examine difference in predictors of restraint use on each category of lands. The population was further stratified by age -0 to 12 years and 13 to 19 years—as seating position recommendations/guidelines exist and vary by age [23,24]. Variables such as roadway characteristics with large quantities of missing data are reported but not included in the multivariable models. Race and ethnicity were not included in the multivariable models because the variable was only reported for drivers who died. All analyses were conducted using SAS 9.4 (SAS Institute, Inc., Cary, NC, USA) [25].

\section{Results}

The study population consisted of 127,747 passengers aged 0 to 19 years being driven in 76,428 passenger vehicles. Of those, $126,080(98.7 \%)$ crashed outside federally designated Indian lands and 1667 (1.3\%) on Indian lands.

Restraint use increased from 2000 to 2014 on both Indian and non-Indian lands, with passengers and drivers on Indian lands consistently showing lower restraint use (Figure 2a).

Among those with known restraint status, $36.8 \%$ of the drivers on Indian lands were restrained, compared to $67.8 \%$ on non-Indian lands $(p<0.0001)$. One third of $(33.1 \%)$ of the passengers on Indian lands were restrained, compared to $59.3 \%$ on non-Indian lands $(p<0.0001)$. Passenger restraint status depended more on whether the vehicle was on Indian lands at the time of the crash and less on other covariates (Figure $2 \mathrm{~b}, \mathrm{c}$ ). Drivers who were positive for drugs or alcohol were more likely to have unrestrained passengers on both Indian and non-Indian lands (Figure $2 b$ ), and passengers of pickup trucks for both Indian lands and non-Indian lands were less likely to be restrained (Figure 2c). Young passengers on Indian lands were less likely to conform to NHTSA rear-seating guidelines (Figure 2d).

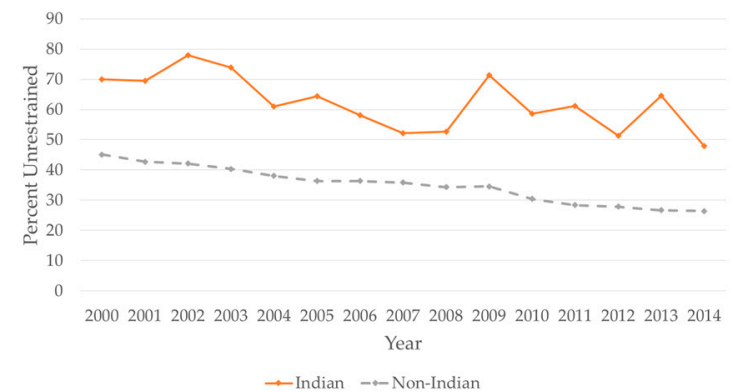

(a)

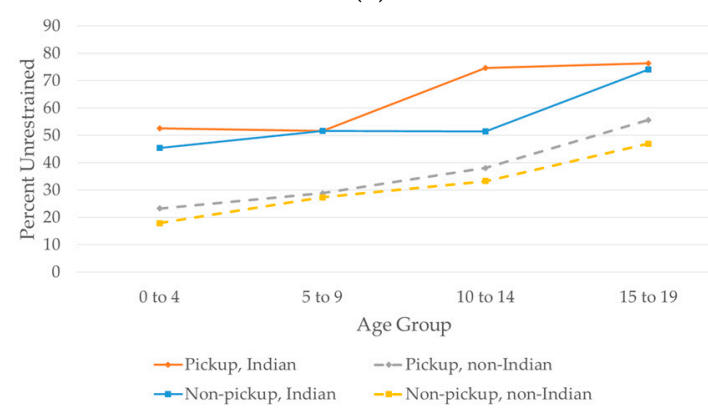

(c)

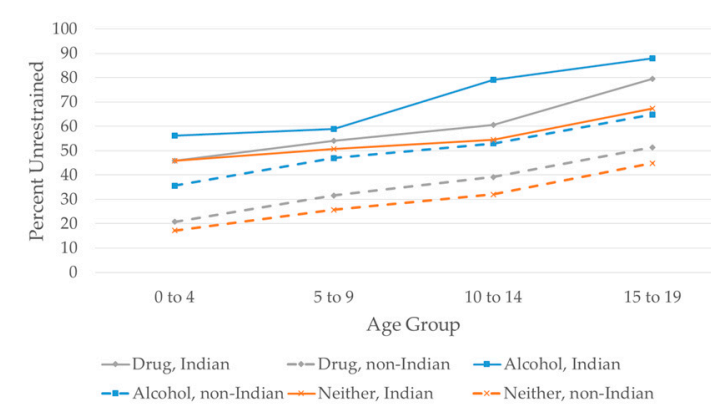

(b)

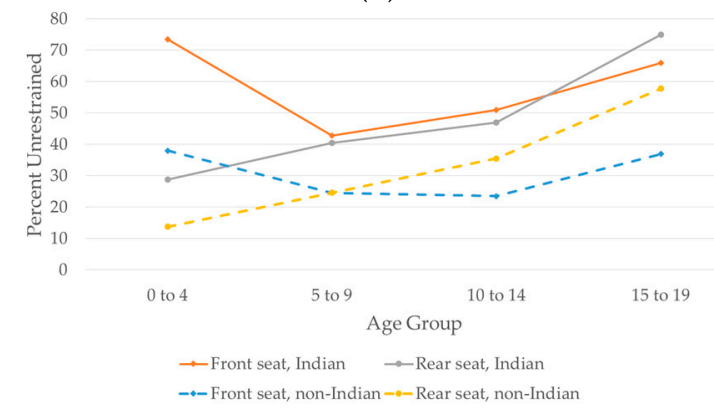

(d)

Figure 2. Percentage of unrestrained passengers aged 0-19 years old by different factors on Indian lands compared to non-Indian lands: (a) Percentage of unrestrained passengers by year; (b) Percentage of unrestrained passengers for driver drug and alcohol use; (c) Percentage of unrestrained passengers for pickup trucks and non-pickup trucks; and (d) Percentage of unrestrained passengers for front seat and rear seat. 


\subsection{Driver Characteristics}

Drivers of vehicles crashing on Indian lands differed from those of non-Indian land drivers by age, gender, belt status, injury severity, license validity, race and ethnicity and previous moving violations (Table 1). The majority of drivers who crashed and died on Indian lands compared to non-Indian lands were reported to be Native American (64.7\% vs. $2.0 \%, p<0.0001)$. Driver mortality was higher on Indian lands compared to non-Indian lands $(34.2 \%$ vs. $29.2 \%, p<0.0001)$ as was passenger injury $(60.4 \%$ vs. $55.0 \%, p<0.0001)$ (Table 1 ).

Drivers crashing on Indian lands were more than twice as likely to have an invalid license compared to those on non-Indian lands ( $35.7 \%$ vs. $15.8 \%, p<0.0001)$. Of the $39.2 \%$ of drivers on Indian lands who were tested for drugs or alcohol, none tested negative $(p<0.0001)$. Drivers of vehicles crashing on Indian lands were less likely to be restrained and were more likely to die as a result of the crash.

\subsection{Passenger Characteristics}

There were no significant differences in the age of passengers between Indian lands and non-Indian lands. Passengers were less likely to be restrained on Indian lands and were more likely to be injured than on non-Indian lands. Passengers of crashes on Indian lands were more likely to be seated in areas of the vehicle other than usual passenger seats (Table 1).

Table 1. Characteristics of crashes on federally recognized Indian lands and non-Indian lands with an infant, child and teen passenger aged 0 to 19 years, FARS 2000-2014.

\begin{tabular}{|c|c|c|c|c|}
\hline Variables & $\begin{array}{c}\text { Indian Lands, } n \\
(\%) *\end{array}$ & $\begin{array}{c}\text { Non-Indian Lands, } n \\
(\%) *\end{array}$ & Total * & $\begin{array}{l}\text { Chi-Square } \\
(p-\text { Value })\end{array}$ \\
\hline Total & $1667(1.30)$ & $126,080(98.70)$ & 127,747 & \\
\hline Driver Characteristics & $868(1.14)$ & $75,359(98.86)$ & 76,227 & \\
\hline Driver age (years) & & & & $182.2(<0.0001)$ \\
\hline$<20$ & $255(29.38)$ & $22,242(29.51)$ & $22,497(29.51)$ & \\
\hline 20 to 44 & $490(56.45)$ & $41,523(55.10)$ & $42,013(55.12)$ & \\
\hline 45 to 64 & $87(10.02)$ & $9607(12.75)$ & $9694(12.72)$ & \\
\hline$\geq 65$ & $11(1.27)$ & $1760(2.34)$ & $1771(2.32)$ & \\
\hline Unknown & $25(2.88)$ & $227(0.30)$ & $252(0.33)$ & \\
\hline Driver gender & & & & $85.8(<0.0001)$ \\
\hline Male & $502(57.84)$ & $44,716(59.34)$ & $45,218(59.32)$ & \\
\hline Female & $354(40.78)$ & $30,537(40.52)$ & $30,891(40.53)$ & \\
\hline Unknown & $12(1.38)$ & $106(0.14)$ & $118(0.15)$ & \\
\hline Driver belt status & & & & $392.7(<0.0001)$ \\
\hline Restrained & $319(36.75)$ & 51,065 (67.76) & $51,384(67.41)$ & \\
\hline Unrestrained & $416(47.93)$ & $19,642(26.06)$ & $20,058(26.31)$ & \\
\hline Unknown & $133(15.32)$ & 4652 (6.16) & $4785(6.28)$ & \\
\hline Injury severity & & & & $127.4(<0.0001)$ \\
\hline Died & $297(34.22)$ & $22,013(29.21)$ & $22,310(29.27)$ & \\
\hline Injured & $423(48.73)$ & $36,780(48.81)$ & $37,203(48.81)$ & \\
\hline Not injured & $126(14.52)$ & $16,283(21.61)$ & $16,409(21.53)$ & \\
\hline Unknown & $22(2.53)$ & $283(0.37)$ & $305(0.40)$ & \\
\hline License validity & & & & $398.2(<0.0001)$ \\
\hline Valid & $504(57.53)$ & $62,305(82.47)$ & $62,809(82.18)$ & \\
\hline Invalid & $313(35.73)$ & $11,909(15.76)$ & $12,222(15.99)$ & \\
\hline Unknown & $59(6.74)$ & 1338 (1.77) & $1397(1.83)$ & \\
\hline $\begin{array}{l}\text { Drug or alcohol tests, } \\
\text { tested only }\end{array}$ & & & & $1.5(0.2270)$ \\
\hline Tested, negative & $0(0.00)$ & $93(0.43)$ & $93(0.42)$ & \\
\hline Tested, positive & $340(100.00)$ & 21,661 (99.57) & $22,001(99.58)$ & \\
\hline
\end{tabular}


Table 1. Cont.

\begin{tabular}{|c|c|c|c|c|}
\hline Variables & $\begin{array}{l}\text { Indian Lands, } n \\
(\%)^{*}\end{array}$ & $\begin{array}{c}\text { Non-Indian Lands, } n \\
(\%)^{*}\end{array}$ & Total * & $\begin{array}{l}\text { Chi-Square } \\
\text { ( } p \text {-Value) }\end{array}$ \\
\hline $\begin{array}{c}\text { Race in death certificate, } \\
\text { w/o N/A }\end{array}$ & & & & $4258.0(<0.0001)$ \\
\hline White, non-Hispanic & $47(15.82)$ & $12,296(55.85)$ & $12,343(55.32)$ & \\
\hline Black, non-Hispanic & $1(0.34)$ & $2636(11.97)$ & $2637(11.82)$ & \\
\hline Hispanic, White or Black & $33(11.11)$ & $3692(16.77)$ & $3725(16.70)$ & \\
\hline Native American & $192(64.65)$ & $431(1.96)$ & $623(2.79)$ & \\
\hline Asian or Pacific Islander & $1(0.34)$ & $473(2.15)$ & $474(2.12)$ & \\
\hline Multiple/Other & $0(0.00)$ & $238(1.08)$ & $238(1.07)$ & \\
\hline Unknown & $23(7.74)$ & 2249 (10.22) & $2272(10.18)$ & \\
\hline \multicolumn{2}{|c|}{ Previous moving violation combined } & & & $305.5(<0.0001)$ \\
\hline Yes & $207(23.63)$ & $21,792(28.84)$ & $21,999(28.78)$ & \\
\hline No & $548(62.56)$ & $51,342(67.96)$ & $51,890(67.89)$ & \\
\hline Unknown & $121(13.81)$ & $2418(3.20)$ & $2539(3.32)$ & \\
\hline $\begin{array}{c}\text { Passenger } \\
\text { Characteristics }\end{array}$ & $1667(1.30)$ & $126,080(98.70)$ & 127,747 & \\
\hline Passenger age (years) & & & & $8.3(0.1405)$ \\
\hline 0 to 2 & $173(10.38)$ & $13,526(10.73)$ & 13,699 (10.72) & \\
\hline 3 to 8 & $323(19.38)$ & $26,735(21.20)$ & $27,058(21.18)$ & \\
\hline 9 to 12 & $217(13.02)$ & $17,245(13.68)$ & $17,462(13.67)$ & \\
\hline 13 to 14 & $176(10.56)$ & $11,383(9.03)$ & $11,559(9.05)$ & \\
\hline 15 to 17 & $431(25.85)$ & $32,022(25.40)$ & $32,453(25.40)$ & \\
\hline 18 to 19 & 347 (20.82) & 25,169 (19.96) & $25,516(19.97)$ & \\
\hline Passenger belt status & & & & $470.3(<0.0001)$ \\
\hline Restrained & $551(33.05)$ & 74,764 (59.30) & $75,315(58.96)$ & \\
\hline Unrestrained & 919 (55.13) & $42,892(34.02)$ & $43,811(34.40)$ & \\
\hline Unknown & $197(11.82)$ & $8424(6.68)$ & $8621(6.75)$ & \\
\hline Injury severity & & & & $79.7(<0.0001)$ \\
\hline Died & $383(22.98)$ & $27,869(22.10)$ & $28,252(22.12)$ & \\
\hline Injured & $1006(60.35)$ & $69,296(54.96)$ & $70,302(55.03)$ & \\
\hline Not injured & $264(15.84)$ & $28,679(22.75)$ & $28,943(22.66)$ & \\
\hline Unknown & $14(0.84)$ & $236(0.19)$ & $250(0.20)$ & \\
\hline Seating position & & & & $531.2(<0.0001)$ \\
\hline Front & $514(30.83)$ & $44,751(35.49)$ & $45,265(35.43)$ & \\
\hline Rear side & $598(35.87)$ & $56,291(44.65)$ & $56,889(44.53)$ & \\
\hline Rear middle & $193(11.58)$ & $14,948(11.86)$ & $15,141(11.85)$ & \\
\hline Other & $94(5.64)$ & $4394(3.49)$ & $4488(3.51)$ & \\
\hline Unknown & $268(16.08)$ & $5696(4.52)$ & $5964(4.67)$ & \\
\hline Vehicle Characteristics & $876(1.15)$ & $75,552(98.85)$ & 76,428 & \\
\hline Vehicle model year & & & & $126.1(<0.0001)$ \\
\hline$<1994$ & $240(27.40)$ & $18,029(23.86)$ & $18,269(23.90)$ & \\
\hline 1994-1997 & 189 (21.58) & $16,435(21.75)$ & $16,624(21.75)$ & \\
\hline 1998-2004 & $328(37.44)$ & 31,118 (41.19) & $31,446(41.14)$ & \\
\hline 2005-2008 & $91(10.39)$ & 7707 (10.20) & $7798(10.20)$ & \\
\hline 2009-2011 & $7(0.80)$ & $1427(1.89)$ & $1434(1.88)$ & \\
\hline 2012-2014 & $8(0.91)$ & $741(0.98)$ & $749(0.98)$ & \\
\hline Unknown & $13(1.48)$ & $95(0.13)$ & $108(0.14)$ & \\
\hline Model type & & & & $49.1(<0.0001)$ \\
\hline Passenger cars & $386(44.06)$ & 39,025 (51.65) & $39,411(51.57)$ & \\
\hline SUV & 177 (20.21) & 16,419 (21.73) & $16,596(21.71)$ & \\
\hline Vans & $88(10.05)$ & 7273 (9.63) & 7361 (9.63) & \\
\hline Pickups & $225(25.68)$ & 12,835 (16.99) & $13,060(17.09)$ & \\
\hline Crash Characteristics & $876(1.15)$ & $75,552(98.85)$ & 76,428 & \\
\hline Day/night & & & & $22.4(<0.0001)$ \\
\hline Day & $404(46.12)$ & $38,552(51.03)$ & $38,956(50.97)$ & \\
\hline Night & 289 (32.99) & $25,500(33.75)$ & $25,789(33.74)$ & \\
\hline Unknown & $183(20.89)$ & $11,500(15.22)$ & $11,683(15.29)$ & \\
\hline Weekday/weekend & & & & $22.6(<0.0001)$ \\
\hline Weekday & $483(55.14)$ & $43,991(58.23)$ & $44,474(58.19)$ & \\
\hline Weekend & $389(44.41)$ & $31,513(41.71)$ & $31,902(41.74)$ & \\
\hline Unknown & $4(0.46)$ & $48(0.06)$ & $52(0.07)$ & \\
\hline
\end{tabular}


Table 1. Cont.

\begin{tabular}{|c|c|c|c|c|}
\hline Variables & $\begin{array}{l}\text { Indian Lands, } n \\
(\%)^{*}\end{array}$ & $\begin{array}{c}\text { Non-Indian Lands, } n \\
(\%)^{*}\end{array}$ & Total * & $\begin{array}{l}\text { Chi-Square } \\
(p \text {-Value })\end{array}$ \\
\hline Rollover & & & & $318.2(<0.0001)$ \\
\hline Yes & $474(54.11)$ & $20,458(27.08)$ & 20,932 (27.39) & \\
\hline No & $402(45.89)$ & $55,094(72.92)$ & $55,496(72.61)$ & \\
\hline Ejected & & & & $328.7(<0.0001)$ \\
\hline Yes & 199 (22.93) & 8377 (11.12) & $8576(11.25)$ & \\
\hline No & $635(73.16)$ & $66,627(88.41)$ & $67,262(88.24)$ & \\
\hline Unknown & $34(3.92)$ & $355(0.47)$ & $389(0.51)$ & \\
\hline Rollover or ejection & & & & $269.9(<0.0001)$ \\
\hline Yes & $502(57.31)$ & $24,278(32.13)$ & $24,780(32.42)$ & \\
\hline No & $362(41.32)$ & $50,899(67.37)$ & $51,261(67.07)$ & \\
\hline Unknown & $12(1.37)$ & $375(0.50)$ & $387(0.51)$ & \\
\hline Manner of collision & & & & $176.2(<0.0001)$ \\
\hline Non-collision & $555(63.36)$ & $33,642(44.53)$ & $34,197(44.14)$ & \\
\hline Rear-end & $47(5.37)$ & $5946(7.87)$ & $5993(7.84)$ & \\
\hline Head-on & $116(13.24)$ & $10,263(13.58)$ & $10,379(13.58)$ & \\
\hline Angle & 122 (13.93) & $22,759(30.12)$ & $22,881(29.94)$ & \\
\hline Sideswipe & $24(2.74)$ & $2561(3.39)$ & $2585(3.38)$ & \\
\hline Other & $5(0.57)$ & $265(0.35)$ & $270(0.35)$ & \\
\hline Unknown & $7(0.80)$ & $116(0.15)$ & $123(0.16)$ & \\
\hline Roadway Characteristics & $876(1.15)$ & $75,552(98.85)$ & 76,428 & \\
\hline Number of lanes & & & & $65.9(<0.0001)$ \\
\hline Non-trafficway & $0(0.00)$ & $76(0.10)$ & $76(0.10)$ & \\
\hline One & $4(0.46)$ & $196(0.26)$ & $200(0.26)$ & \\
\hline Two & $209(23.86)$ & $12,540(16.60)$ & $12,749(16.68)$ & \\
\hline Three & $3(0.34)$ & $1613(2.13)$ & $1616(2.11)$ & \\
\hline Four & $7(0.80)$ & 2467 (3.27) & $2474(3.24)$ & \\
\hline Five & $2(0.23)$ & $481(0.64)$ & $483(0.63)$ & \\
\hline Six or more & $0(0.00)$ & $353(0.47)$ & $353(0.46)$ & \\
\hline Unknown & $651(74.32)$ & $57,826(76.54)$ & $58,477(76.51)$ & \\
\hline $\begin{array}{c}\text { Trafficway } \\
\text { (one/two-way) }\end{array}$ & & & & $45.0(<0.0001)$ \\
\hline Non-trafficway & $0(0.00)$ & $76(0.10)$ & $76(0.10)$ & \\
\hline One-way & $3(0.34)$ & $187(0.25)$ & $190(0.25)$ & \\
\hline Two-way, divided & $36(4.11)$ & $6118(8.10)$ & $6154(8.05)$ & \\
\hline Two-way, not divided & $186(21.23)$ & $11,190(14.81)$ & $11,376(14.88)$ & \\
\hline Other & $0(0.00)$ & $187(0.25)$ & $187(0.24)$ & \\
\hline Unknown & $651(74.32)$ & $57,794(76.50)$ & $58,445(76.47)$ & \\
\hline Traffic devices and signs & & & & $40.8(<0.0001)$ \\
\hline No controls & $210(23.97)$ & $13,728(18.17)$ & $13,938(18.27)$ & \\
\hline Traffic signals & $0(0.00)$ & $1855(2.46)$ & $1855(2.43)$ & \\
\hline Regulatory signs & $16(1.83)$ & $2049(2.71)$ & $2065(2.70)$ & \\
\hline Other & $2(0.23)$ & $145(0.19)$ & $147(0.19)$ & \\
\hline Unknown & $648(73.97)$ & $57,775(76.47)$ & $58,423(76.44)$ & \\
\hline Route signing & & & & $417.8(<0.0001)$ \\
\hline Interstate & $59(7.13)$ & $10,929(14.53)$ & $10,988(14.45)$ & \\
\hline Highway & $416(50.30)$ & $30,547(40.62)$ & $30,963(40.72)$ & \\
\hline Country road & $180(21.77)$ & $14,976(19.91)$ & $15,156(19.93)$ & \\
\hline Local street & $29(3.51)$ & $15,102(20.08)$ & $15,131(19.90)$ & \\
\hline Other & $143(17.29)$ & $3650(4.85)$ & $3793(4.99)$ & \\
\hline $\begin{array}{l}\text { Traffic control device } \\
\text { functioning }\end{array}$ & & & & $35.4(<0.0001)$ \\
\hline Not functioning properly & $0(0.00)$ & $20(0.03)$ & $20(0.03)$ & \\
\hline Functioning properly & $17(1.94)$ & $3999(5.29)$ & $4016(5.25)$ & \\
\hline No controls & $210(23.97)$ & $13,728(18.17)$ & $13,938(18.24)$ & \\
\hline Unknown & 649 (74.09) & $57,805(76.51)$ & $58,454(76.48)$ & \\
\hline
\end{tabular}

In the multivariable adjusted model, passengers on Indian lands were less likely to be restrained on Indian lands than on non-Indian lands (Table 2). The effect of Indian land crash site on likelihood of being restrained is more pronounced for passengers aged $0-12(\mathrm{OR}=0.535,95 \% \mathrm{CI} 0.416,0.715)$ 
than for passengers aged $13-19$ years $(\mathrm{OR}=0.635,95 \%$ CI $0.500,0.807)$, controlling for driver and vehicle characteristics.

Table 2. Adjusted ORs (95\% CIs) for infant, child and teen being restrained in the states with federally recognized Indian lands, FARS 2000-2014, categorized by passenger ages 0-12 and 13-19 years.

\begin{tabular}{|c|c|c|}
\hline Variables & 0-12 Adjusted, Restrained & 13-19 Adjusted, Restrained \\
\hline & $n=44,459$ & $n=50,565$ \\
\hline \multicolumn{3}{|l|}{ Driver Characteristics } \\
\hline \multicolumn{3}{|l|}{ Driver age (years) } \\
\hline$<20$ & Ref & Ref \\
\hline 20 to 44 & $2.023(1.798,2.276)$ & $1.272(1.208,1.340)$ \\
\hline 45 to 64 & $1.840(1.601,2.115)$ & $1.840(1.687,2.008)$ \\
\hline$\geq 65$ & $2.234(1.780,2.803)$ & $2.063(1.667,2.554)$ \\
\hline \multicolumn{3}{|l|}{ Driver restraint use } \\
\hline Not restrained & Ref & Ref \\
\hline Restrained & $7.759(7.228,8.330)$ & $10.607(10.046,11.200)$ \\
\hline \multicolumn{3}{|l|}{ Driver gender } \\
\hline Male & Ref & Ref \\
\hline Female & $1.021(0.958,1.088)$ & $1.074(1.019,1.132)$ \\
\hline \multicolumn{3}{|l|}{ License validity } \\
\hline Invalid & Ref & Ref \\
\hline Valid & $2.098(1.930,2.281)$ & $1.500(1.409,1.598)$ \\
\hline \multicolumn{3}{|l|}{ Drug or alcohol tests } \\
\hline Tested, negative & Ref & Ref \\
\hline Tested, negative & $1.464(0.609,3.520)$ & $0.870(0.487,1.555)$ \\
\hline Not tested & $1.699(0.707,4.084)$ & $1.087(0.608,1.943)$ \\
\hline \multicolumn{3}{|c|}{ Vehicle Characteristics } \\
\hline \multicolumn{3}{|l|}{ Model type } \\
\hline Passenger cars & Ref & Ref \\
\hline SUV & $0.820(0.758,0.887)$ & $0.742(0.696,0.790)$ \\
\hline Vans & $0.695(0.631,0.766)$ & $0.622(0.566,0.683)$ \\
\hline Pickups & $0.777(0.705,0.855)$ & $0.754(0.704,0.807)$ \\
\hline \multicolumn{3}{|l|}{ Crash Characteristics } \\
\hline \multicolumn{3}{|c|}{ Indian land status of the crash site } \\
\hline Non-Indian land & Ref & Ref \\
\hline Indian land & $0.535(0.416,0.715)$ & $0.635(0.500,0.807)$ \\
\hline
\end{tabular}

\subsubsection{Passenger Restraint Use for Indian Lands}

In the multivariable adjusted model, passengers across all ages from 0 to 19 years were more likely to be restrained when the driver was restrained (Table 3), controlling for driver age, gender, license validity, drug or alcohol test results and vehicle model type. The odds of passengers being restrained when the driver was restrained is greater for passengers aged 13-19 compared to passengers aged 0-12 years (Figure 3a). Passengers aged 0-12 were more likely to be restrained when the driver had a valid license, but this effect was not significant for passengers aged 13-19 years (Figure 3b). Passengers aged 0-12 years were more likely to be belted with drivers aged 20-44, and passengers aged 13-19 were more likely to be belted with drivers aged 45-64 years. 
Table 3. Unadjusted and adjusted ORs (95\% CIs) for infant, child and teen restraint status on Indian lands in the states with federally recognized Indian lands, FARS 2000-2014, divided by passenger ages 0-12 and 13-19 years.

\begin{tabular}{|c|c|c|c|c|}
\hline Variables & $\begin{array}{l}\text { 0-12 Unadjusted, } \\
\text { Restrained }\end{array}$ & $\begin{array}{l}\text { 0-12 Adjusted, } \\
\text { Restrained }\end{array}$ & $\begin{array}{l}\text { 13-19 Unadjusted, } \\
\text { Restrained }\end{array}$ & $\begin{array}{l}\text { 13-19 Adjusted, } \\
\text { Restrained }\end{array}$ \\
\hline & $n=713$ & $n=520$ & $n=954$ & $n=628$ \\
\hline \multicolumn{5}{|l|}{ Driver Characteristics } \\
\hline \multicolumn{5}{|l|}{ Driver age (years) } \\
\hline$<20$ & Ref & Ref & Ref & Ref \\
\hline 20 to 44 & $3.726(1.624,8.549)$ & $2.396(0.826,6.951)$ & $1.618(1.039,2.519)$ & $1.273(0.716,2.265)$ \\
\hline 45 to 64 & $4.895(1.740,13.775)$ & $1.225(0.324,4.629)$ & $12.090(5.091,28.709)$ & $4.763(1.464,15.495)$ \\
\hline$\geq 65$ & $1.899(0.184,19.636)$ & $0.286(0.012,6.644)$ & $10.558(1.280,87.603)$ & $1.904(0.040,91.206)$ \\
\hline \multicolumn{5}{|l|}{ Driver restraint use } \\
\hline Not restrained & Ref & Ref & Ref & Ref \\
\hline Restrained & $8.496(4.989,14.468)$ & $6.548(3.262,13.143)$ & $22.887(14.029,37.336)$ & $\begin{array}{l}13.885(7.702 \\
25.033)\end{array}$ \\
\hline \multicolumn{5}{|l|}{ Driver gender } \\
\hline Male & Ref & Ref & Ref & Ref \\
\hline Female & $0.913(0.556,1.500)$ & $0.711(0.382,1.324)$ & $1.693(1.109,2.586)$ & $1.300(0.742,2.278)$ \\
\hline \multicolumn{5}{|l|}{ License validity } \\
\hline Invalid & Ref & Ref & Ref & Ref \\
\hline Valid & $5.894(3.314,10.484)$ & $2.662(1.249,5.674)$ & $3.929(2.512,6.146)$ & $1.738(0.966,3.129)$ \\
\hline \multicolumn{5}{|l|}{ Drug or alcohol tests } \\
\hline Tested, positive ${ }^{1}$ & Ref & Ref & Ref & Ref \\
\hline Tested, negative & - & - & - & - \\
\hline Not tested & $1.577(0.936,2.658)$ & $1.226(0.646,2.325)$ & $4.426(2.808,6.977)$ & $1.563(0.827,2.956)$ \\
\hline \multicolumn{5}{|l|}{$\begin{array}{c}\text { Vehicle } \\
\text { Characteristics }\end{array}$} \\
\hline \multicolumn{5}{|l|}{ Model type } \\
\hline Passenger cars & Ref & Ref & Ref & Ref \\
\hline SUVs & $0.746(0.395,1.411)$ & $0.937(0.422,2.081)$ & $1.300(0.755,2.238)$ & $0.489(0.229,1.046)$ \\
\hline Vans & $1.023(0.475,2.204)$ & $1.103(0.425,2.862)$ & $2.672(1.232,5.795)$ & $0.932(0.344,2.523)$ \\
\hline Pickups & $0.532(0.279,1.014)$ & $0.553(0.246,1.244)$ & $0.966(0.581,1.606)$ & $0.604(0.307,1.185)$ \\
\hline
\end{tabular}

${ }^{1}$ Positive test result was made the reference value here because no driver was tested negative for drugs or alcohols on Indian lands.

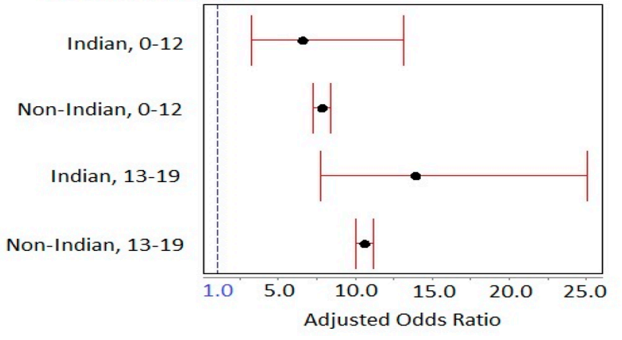

(a)

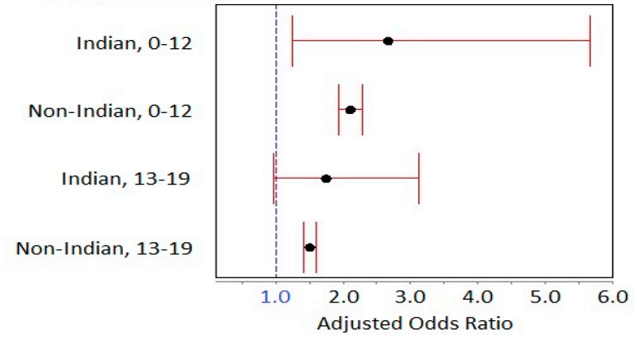

(b)

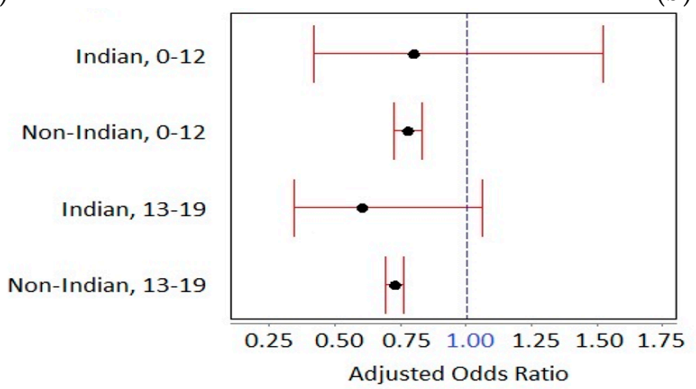

(c)

Figure 3. Independent predictors of passenger (aged 0-19 years) restraint use for Indian lands and non-Indian lands by age group, FARS 2000-2014: (a) Driver restraint use as an independent predictor of passenger (aged 0-19 years) restraint use (Adjusted for driver age, gender, license validity, drug/alcohol status and vehicle model type); (b) Driver valid license as an independent predictor of passenger (aged 0-19 years) restraint use (Adjusted for driver age, gender, restraint status, drug/alcohol status and vehicle model type); (c) Vehicle type as an independent predictor of passenger (aged 0-19 years) restraint use (Adjusted for driver age, gender, restraint status, license validity and drug/alcohol status). 
Passengers were generally less likely to be restrained in vehicles other than passenger cars (SUVs, vans, and pickups) (Figure 3c). Driver gender was not a significant predictor of passenger belt status in either unadjusted and adjusted models; a positive drug or alcohol test result was significant in unadjusted models but no longer significant in adjusted models.

\subsubsection{Passenger Restraint Use for Non-Indian Lands}

Passengers across all ages from 0 to 19 years were more likely to be restrained when the driver was restrained and had a valid license, but with smaller odds ratios compared to Indian lands, controlling for driver age, gender, license validity, drug or alcohol test results and vehicle model type (Table 4). Again, the odds of passengers being restrained when the driver was restrained was greater for passengers aged 13-19 compared to passengers aged 0-12 years (Figure 3a). Driver gender was a significant predictor for both unadjusted and adjusted models in passengers aged 0-12 years, but not for adjusted models of teen passengers. Drug or alcohol test results were significant only in unadjusted models, and only between those not tested and those who tested negative. Passengers were less likely to be belted in vehicles other than passenger cars (SUVs, vans, and pickups) (Figure 3c). Unadjusted comparisons between vans and passenger cars for passenger restraint status show a reversal of effect when adjusted for other variables.

Table 4. Unadjusted and adjusted ORs (95\% CIs) for infant, child and teen restraint status on non-Indian lands in the states with federally recognized Indian lands, FARS 2000-2014, categorized by passenger ages $0-12$ and $13-19$ years.

\begin{tabular}{|c|c|c|c|c|}
\hline Variables & $\begin{array}{l}\text { 0-12 Unadjusted, } \\
\text { Restrained }\end{array}$ & $\begin{array}{l}\text { 0-12 Adjusted, } \\
\text { Restrained }\end{array}$ & $\begin{array}{l}\text { 13-19 Unadjusted, } \\
\text { Restrained }\end{array}$ & $\begin{array}{l}\text { 13-19 Adjusted, } \\
\text { Restrained }\end{array}$ \\
\hline & $n=57,506$ & $n=43,939$ & $n=68,574$ & $n=49,937$ \\
\hline \multicolumn{5}{|c|}{ Driver Characteristics } \\
\hline \multicolumn{5}{|l|}{ Driver age (years) } \\
\hline$<20$ & Ref & Ref & Ref & Ref \\
\hline 20 to 44 & $2.276(2.063,2.511)$ & $2.013(1.787,2.267)$ & $1.228(1.179,1.280)$ & $1.272(1.208,1.339)$ \\
\hline 45 to 64 & $2.367(2.110,2.657)$ & $1.842(1.601,2.119)$ & $2.701(2.518,2.898)$ & $1.829(1.676,1.996)$ \\
\hline$\geq 65$ & $3.050(2.524,3.686)$ & $2.249(1.790,2.825)$ & $3.157(2.649,3.763)$ & $2.066(1.669,2.558)$ \\
\hline \multicolumn{5}{|l|}{ Driver restraint use } \\
\hline Not restrained & Ref & Ref & Ref & Ref \\
\hline Restrained & $8.509(8.002,9.049)$ & $7.799(7.261,8.378)$ & $12.459(11.879,13.068)$ & $10.565(10.004,11.159)$ \\
\hline \multicolumn{5}{|l|}{ Driver gender } \\
\hline Male & Ref & Ref & Ref & Ref \\
\hline Female & $1.170(1.111,1.233)$ & $1.026(0.962,1.093)$ & $1.593(1.527,1.661)$ & $1.074(1.018,1.132)$ \\
\hline \multicolumn{5}{|l|}{ License validity } \\
\hline Invalid & Ref & Ref & Ref & Ref \\
\hline Valid & $2.608(2.431,2.798)$ & $2.097(1.928,2.281)$ & $2.036(1.935,2.143)$ & $1.495(1.403,1.592)$ \\
\hline \multicolumn{5}{|l|}{ Drug or alcohol tests } \\
\hline Tested, negative & Ref & Ref & Ref & Ref \\
\hline Tested, positive & $1.219(0.559,2.658)$ & $1.462(0.608,3.514)$ & $0.985(0.592,1.639)$ & $0.861(0.482,1.535)$ \\
\hline Not tested & $2.142(0.983,4.667)$ & $1.695(0.706,4.073)$ & $2.014(1.210,3.350)$ & $1.074(0.602,1.916)$ \\
\hline \multicolumn{5}{|l|}{$\begin{array}{c}\text { Vehicle } \\
\text { Characteristics }\end{array}$} \\
\hline \multicolumn{5}{|l|}{ Model type } \\
\hline Passenger cars & Ref & Ref & Ref & Ref \\
\hline SUVs & $0.938(0.878,1.003)$ & $0.819(0.757,0.887)$ & $0.934(0.887,0.984)$ & $0.744(0.698,0.793)$ \\
\hline Vans & $0.968(0.892,1.050)$ & $0.691(0.627,0.762)$ & $1.128(1.046,1.217)$ & $0.619(0.563,0.680)$ \\
\hline Pickups & $0.766(0.708,0.830)$ & $0.784(0.711,0.864)$ & $0.700(0.663,0.739)$ & $0.755(0.705,0.808)$ \\
\hline
\end{tabular}




\subsection{Vehicle Characteristics}

Vehicle model year and model type were associated with Indian land status of the crash site. Indian lands had a higher proportion of pre-1994 vehicles than non-Indian lands $(p<0.0001)$, and a higher proportion of pickup trucks than non-Indian lands $(p<0.0001)$ (Table 1$)$.

\subsection{Crash Characteristics}

Crash time (day/night) and day (weekday/weekend), as well as rollover, ejection and manner of collision were all significantly associated with Indian land status. Vehicles that crashed on Indian lands were twice as likely to experience rollover ( $54.1 \%$ vs. $27.1 \%, p<0.0001)$, and drivers of vehicles on Indian lands were twice as likely to be ejected upon collision $(22.9 \%$ vs. $11.1 \%, p<0.0001)$ (Table 1$)$.

Single motor vehicle crashes consisted of $63.4 \%$ of all crashes on Indian lands, compared to $44.5 \%$ on non-Indian lands. Crashes in angle with other motor vehicles consisted of $30.1 \%$ of all crashes on non-Indian lands, compared to $13.9 \%$ on Indian lands $(p<0.0001)$ (Table 1$)$.

\subsection{Roadway Characteristics}

Number of lanes, trafficway (one/two-way), traffic devices and signs and the functioning status of traffic control devices around the crash site had more than $70 \%$ of the data missing for both Indian lands and non-Indian lands. Analysis of reported data showed that crashes occurred mostly on highways $(50.3 \%)$ and country roads $(21.8 \%)$ for those on Indian lands, and on highways $(40.6 \%)$ and local streets $(20.1 \%)$ for those on non-Indian lands (Table 1$)$.

\section{Discussion}

In many countries that also contain native population jurisdictions such as is found in the U.S., particular consideration should be given to examination and ongoing monitoring of the health impacts in geographic areas where motor vehicle laws and regulations differ from best practices. Examining these areas separately and providing the findings to local entities can stimulate local culturally-sensitive engagement in setting intervention priorities and in designing culturally appropriate solutions and interventions, including crafting of laws or regulations, aimed at lowering the burden of motor vehicle injury in the vulnerable populations that tend to live in these areas.

The strongest predictor of a pediatric passenger being unrestrained was whether the crash occurred on Indian lands compared to non-Indian lands in states containing these lands. Passenger restraint depended more on whether the vehicle crashed on Indian lands than on other covariates. This finding was surprisingly more pronounced in teens than in younger passengers aged 0-12 years.

There were several significant differences in the characteristics of crashes on Indian lands compared to non-Indian lands. Within geographic regions, driver restraint was a highly important factor, a finding consistent with what is generally known $[9,11,15]$. Environmental characteristics appear to play an important role with crashes on Indian lands being more likely to be on two lane roads and to be single vehicle collisions. There was one difference in particular that may have contributed to smaller disparities in mortality on Indian lands than would have been expected given the vast number of characteristics present in higher proportions on Indian lands that are known to contribute to higher mortality. In particular, the higher proportion of pickup trucks to passenger cars is generally associated with heavier vehicles and vehicles in which passengers sit higher and may be more protected than in those in passenger cars. This study did not address how these findings compare when controlling for rural and sparsely populated lands, a factor that should be addressed in future study [26]. The relative importance of some key covariates varied across passenger age categories that deserve further examination.

Our findings are consistent with earlier studies that showed American Indian/Alaskan Native (AI/AN) passengers aged 0-12 years of age are less likely to be in compliance with NHTSA recommended rear seating guidelines [27]. Our study extends these findings by demonstrating 
continued disparities in the noncompliance with guidelines across geographic jurisdictions. This issue is especially alarming, given that a crash on Indian lands is more predictive of being unrestrained for passengers aged 0-12 than for those aged 13-19 years.

Driver belt status was a strong predictor of passenger restraint use for teens on Indian lands where passengers of restrained drivers had overwhelming odds of being restrained compared to those travelling with unrestrained drivers. There was a much larger proportion of drivers unrestrained on Indian lands, and teen passenger restraint status mirrored that of the drivers transporting them [28]. Passengers aged 0-12 years on Indian lands being transported by drivers with valid driver licenses had the highest odds of being restrained. Our finding that the percentage of unrestrained passengers is the highest with drivers who tested positive for alcohol, followed by those tested positive for drugs and those tested positive for neither or who were not tested is consistent with previous studies $[12,13]$.

Rollovers and ejections, previously reported to be associated with higher mortality, were significantly higher on Indian lands, which could have contributed to higher passenger mortality and injury, especially in those who were unrestrained. Furthermore, single vehicle collisions occurred more frequently on Indian lands and were more likely to result in rollover of vehicles than other types of collisions.

This study has limitations. Although this study was limited to the crashes in states with federally recognized Indian lands, there is significant heterogeneity within the Indian land category. We did not have variables in our data set to facilitate investigation of these differences. Our study only investigated passenger restraint status as restrained or unrestrained and did not consider the importance of child restraint types across younger age groups. We did not take holidays into account because holidays vary significantly across cultures, religions and geographic areas within the U.S. and across the geographic areas under study. There are variations in strength of laws and the enforcement of these laws within each Indian nation, as well as social structures that could play a contributing role. Of significance, is the disparities in the proportions of missing data in important variables. We had limited data on travel speed for both jurisdictions that precluded our investigation of this important factor. The issue of missing data was more pronounced for roadway characteristics variables, for which some of the percentages of missing data were as high as three quarters of the study population. Furthermore, percentages of missing data for most variables were consistently higher for crashes on Indian lands than for crashes on non-Indian lands. This suggests the need for improvement in data collection in both jurisdictions but particularly on Indian lands.

\section{Conclusions}

These findings suggest that there are marked differences in crash characteristics between the Indian lands and non-Indian lands with regard to vehicle, passenger, driver and crash characteristics that have been linked to higher MV mortality and morbidity. Infant, child and teen restraint patterns continue to be significantly lower on Indian lands compared to adjacent non-Indian lands. Although restraint use improved over the study period on both Indian and non-Indian lands, disparities remained and failed to narrow. Disparities in data quality between Indian lands and non-Indian lands were an obstacle to assessing several key risk factors. This pattern, in conjunction with differing environmental characteristics, is associated with greater pediatric endangerment on Indian lands compared to non-Indian lands.

Acknowledgments: This analysis was performed by an intern (SO) in fulfillment of a degree requirement for her MS in biostatistics at Columbia University. The contents of the manuscript are the sole responsibility of the authors and do not necessarily represent the official views of the National Highway and Traffic Safety Administration or other data collection agencies. This study received partial support from the National Center for Injury Prevention and Control of the Centers for Disease Control and Prevention (Grant 1R49 CE002096).

Author Contributions: S.O. performed the statistical analyses and preparation of tables and figures and drafted the original manuscript. C.L. contributed to preparation of tables and figures and manuscript preparation. J.P. supervised the internship, statistical analysis, contributed critical content and revision of the manuscript. All authors read and approved the final manuscript. 
Conflicts of Interest: The authors declare no conflict of interest.

\section{References}

1. Murphy, T.; Pokhrel, P.; Worthington, A.; Billie, H.; Sewell, M.; Bill, N. Unintentional injury mortality among American Indians and Alaska Natives in the United States, 1990-2009. Am. J. Public Health 2014, 104, S470-S480. [CrossRef] [PubMed]

2. Centers for Disease Control and Prevention. Web-based Injury Statistics Query and Reporting System (WISQARS). Available online: https:/ / www.cdc.gov/injury/wisqars/ (accessed on 16 June 2016).

3. Pressley, J.C.; Barlow, B.; Kendig, T.; Paneth-Pollak, R. Twenty-year trends in fatal injuries to very young children: The persistence of racial disparities. Pediatrics 2007, 119, e875-e884. [CrossRef] [PubMed]

4. Ernat, J.J.; Knox, J.B.; Wimberly, R.L.; Riccio, A.I. The effects of restraint type on pattern of spine injury in children. J. Pediatr. Orthop. 2016, 36, 594-601. [CrossRef] [PubMed]

5. Johnston, C.; Rivara, F.P.; Soderberg, R. Children in car crashes: Analysis of data for injury and use of restraints. Pediatrics 1994, 93, 960-965. [PubMed]

6. Valent, F.; McGwin, G.; Hardin, W.; Johnston, C.; Rue, L.W. Restraint use and injury patterns among children involved in motor vehicle collisions. J. Trauma Acute Care Surg. 2002, 52, 745-751. [CrossRef]

7. Lapidus, J.A.; Smith, N.H.; Ebel, B.E.; Romero, F.C. Restraint use among northwest American Indian children traveling in motor vehicles. Am. J. Public Health 2005, 95, 1982-1988. [CrossRef] [PubMed]

8. Smith, M.; Berger, L. Assessing community child passenger safety efforts in three northwest tribes. Inj. Prev. 2002, 8, 289-292. [CrossRef] [PubMed]

9. Iragavarapu, V.; Carlson, P.; Schertz, G. Review of Tribal Transportation Safety. Transp. Res. Rec. J. Transp. Res. Board 2015, 2531, 153-160. [CrossRef]

10. Leaf, W.; Solomon, M.G. Safety Belt Use Estimate for Native American Tribal Reservations. Available online: http:/ / ntl.bts.gov/lib /27000/27000/27032/textfinal.pdf (accessed on 21 June 2016).

11. National Highway Traffic Safety Administration. Safety Belt Use Estimate for Native American Tribal Reservations. Available online: https://www.nhtsa.gov/document/safety-belt-use-estimate-nativeamerican-tribal-reservations (accessed on 20 June 2016).

12. Huang, Y.; Liu, C.; Pressley, J.C. Child restraint use and driver screening in fatal crashes involving drugs and alcohol. Pediatrics 2016, 138, e20160319. [CrossRef] [PubMed]

13. Maio, R.F.; Waller, P.F.; Blow, F.C.; Hill, E.M.; Singer, K.M. Alcohol abuse/dependence in motor vehicle crash victims presenting to the emergency department. Acad. Emer. Med. 1997, 4, 256-262. [CrossRef]

14. Agran, P.F.; Anderson, C.L.; Winn, D.G. Factors associated with restraint use of children in fatal crashes. Pediatrics 1998, 102, e39. [CrossRef] [PubMed]

15. Poindexter, K. Fatal Motor Vehicle Crashes on Indian Reservations, 1975-2002. Available online: https: / / crashstats.nhtsa.dot.gov/Api/Public/ViewPublication/809727 (accessed on 18 August 2017).

16. Jim, M.A.; Arias, E.; Seneca, D.S.; Hoopes, M.J.; Jim, C.C.; Johnson, N.J.; Wiggins, C.L. Racial misclassification of American Indians and Alaska Natives by Indian Health Service contract health service delivery area. Am. J. Public Health 2014, 104, S295-S302. [CrossRef] [PubMed]

17. Stevens, J.A.; Dellinger, A.M. Motor vehicle and fall related deaths among older Americans 1990-98: Sex, race, and ethnic disparities. Inj. Prev. 2002, 8, 272-275. [CrossRef] [PubMed]

18. Sugarman, J.R.; Soderberg, R.; Gordon, J.E.; Rivara, F.P. Racial misclassification of American Indians: Its effect on injury rates in Oregon, 1989 through 1990. Am. J. Public Health 1993, 83, 681-684. [CrossRef] [PubMed]

19. National Highway Traffic Safety Administration. Fatality Analysis Reporting System (FARS) Analytical User's Manual 1975-2014. Available online: https://crashstats.nhtsa.dot.gov/Api/Public/Publication/ 812315 (accessed on 18 August 2017).

20. National Conference of State Legislatures. Federal and State Recognized Tribes. Available online: http:/ / www.ncsl.org/research/state-tribal-institute/list-of-federal-and-state-recognized-tribes. aspx\#federal (accessed on 18 August 2017).

21. Ryb, G.E.; Dischinger, P.C.; McGwin, G.; Griffin, R.L. Crash-related mortality and model year: Are newer vehicles safer? In Proceedings of the Annals of Advances in Automotive Medicine Annual Scientific Conference, Paris, France, 3-5 October 2011; Association for the Advancement of Automotive Medicine: Chicago, IL, USA, 2011; p. 113. 
22. Carpenter, D.; Pressley, J.C. Graduated driver license nighttime compliance in US teen drivers involved in fatal motor vehicle crashes. Accid. Anal. Prev. 2013, 56, 110-117. [CrossRef] [PubMed]

23. National Highway Traffic Safety Administration. Car Seat Recommendations for Children. Available online: https: / /www.nhtsa.gov / DOT/NHTSA/Traffic\%20Injury\%20Control/Articles / Associated\%20Files / 4StepsFlyer.pdf (accessed on 18 August 2017).

24. Governors Highway Safety Association. Child Passenger Safety Laws. Available online: http://www.ghsa. org/html/stateinfo/laws/childsafety_laws.html (accessed on 18 August 2017).

25. SAS Institute Inc. Base SAS®9.4 Procedures Guide: Statistical Procedures. Available online: https:/ / support. sas.com/documentation/cdl/en/procstat/66703/PDF/.../procstat.pdf (accessed on 18 August 2017).

26. Beck, L.F.; Downs, J.; Stevens, M.R.; Sauber-Schatz, E.K. Rural and urban differences in passenger-vehicle-Occupant deaths and seat belt use among adults-United States, 2014. MMWR. Surveill. Summ. 2017, 66, 1-13. [CrossRef] [PubMed]

27. Decina, L.E.; Lococo, K.H. Child restraint system use and misuse in six states. Accid. Anal. Prev. 2005, 37, 583-590. [CrossRef] [PubMed]

28. Miller, T.R.; Spicer, R.S.; Lestina, D.C. Who is driving when unrestrained children and teenagers are hurt? Accid. Anal. Prev. 1998, 30, 839-849. [CrossRef]

(C) 2017 by the authors. Licensee MDPI, Basel, Switzerland. This article is an open access article distributed under the terms and conditions of the Creative Commons Attribution (CC BY) license (http:/ / creativecommons.org/licenses/by/4.0/). 